\title{
Syndetic extension of baryon and lepton numbers: Proton decay and long-lived dark matter
}

\author{
Ernest $\mathrm{Ma}^{1}$ and Koji Tsumura, ${ }^{2, *}$ \\ ${ }^{1}$ Physics and Astronomy Department, University of California, Riverside, California 92521, USA \\ ${ }^{2}$ Department of Physics, Kyoto University, Kyoto 606-8502, Japan
}

(Received 26 June 2018; published 27 August 2018)

\begin{abstract}
The well-known baryon and lepton numbers of the standard model of quarks and leptons are extended to include new fermions and bosons in a simple structure with several essential features. The usual heavy right-handed neutrino singlets (for neutrino mass and leptogenesis) are related to the axion which solves the strong $C P$ problem. At the same time, baryon number is broken softly, allowing the proton to decay. Associated with this breaking, a long-lived dark-matter candidate (called the pseudosakharon) emerges. This new insight connects proton decay to a new component of dark matter.
\end{abstract}

DOI: 10.1103/PhysRevD.98.035037

\section{INTRODUCTION}

The standard model (SM) of quarks and leptons is known to have the built-in global $U(1)$ symmetries of baryon number $B$ and lepton number $L$. If new particles are added, their $B$ and $L$ assignments may be chosen judiciously [1] to address a number of outstanding theoretical issues. In the following, it will be shown how a simple extension of the SM, which connects [2-4] the seesaw neutrino mass with the axion decay constant, may also allow a new understanding of the longevity [5] of weak-scale dark matter (DM), i.e., that it is related to proton decay.

If the SM is to be extended, one may want to consider the fundamental issues of (I) nonzero neutrino mass, (II) DM, and (III) strong $C P$ nonconservation in quantum chromodynamics (QCD). A simple connection was proposed thirty years ago [3], where the neutrino mass seesaw anchor scale is identified with the vacuum expectation value of a singlet scalar field which couples anomalously to new very heavy quark singlet fields $\left(\Psi_{L, R}\right)$ as well as the three very heavy right-handed neutrinos $\left(N_{R}\right)$. Together with the wellknown mechanism of leptogenesis [6], this also explains the baryon asymmetry of the Universe. The DM of this model is the invisible axion which is yet to be discovered. However, it is not guaranteed that the axion accounts for all of DM. In fact, the anomalous Peccei-Quinn symmetry [7] which yields the axion [8,9] has in general a residual discrete $Z_{2}$ symmetry [10] which may be relevant for

\footnotetext{
*ko2@gauge.scphys.kyoto-u.ac.jp
}

Published by the American Physical Society under the terms of the Creative Commons Attribution 4.0 International license. Further distribution of this work must maintain attribution to the author(s) and the published article's title, journal citation, and DOI. Funded by SCOAP ${ }^{3}$. weak-scale DM. In that case, the strong $C P$ problem may well be solved by the axion, but the latter may only be a small component of DM, whereas the bulk comes from a weak-scale DM particle, odd under this $Z_{2}$. The axionneutrino connection implies the basic assumption $[11,12]$ $U(1)_{P Q}=U(1)_{L}$, and that $\Psi$ transforms under both $B$ and $L$.

Another theoretical issue is whether or not DM is truly stable, in which case it should be protected by a symmetry, or just a very long-lived particle such as the invisible axion. It is now known that such a DM particle must have a lifetime orders of magnitude longer than the age of the Universe, to avoid disrupting [13] the cosmic microwave background and other astrophysical observations [14]. The only possible exception is for the DM to decay dominantly to neutrinos, which was implemented in a recent model [15] where lepton number becomes a discrete $Z_{3}$ symmetry.

If $\mathrm{DM}$ is not absolutely stable, then its lifetime must be very long. This may be due to the possible unification of matter and DM at a very high scale [5] or that it is somehow related to a known lifetime which is very long. This brings to mind proton decay and in this paper it will be shown how the two may be related.

This paper is organized as follows. In Sec. II, the particle content and the relevant interactions are introduced, which define the unique lepton and baryon numbers of the new particles. In Sec. III, the longevity of the DM is linked to the proton decay. A scenario for thermal freeze out of the DM is also discussed. Summary of our new proposal is given in Sec. IV.

\section{THE MODEL}

The axion-neutrino connection is established using a very heavy colored electroweak singlet quark $\Psi$ and three 
TABLE I. Particle content of model with axion and pseudosakharon.

\begin{tabular}{lccc}
\hline \hline Particle & $S U(3)_{C} \times S U(2)_{L} \times U(1)_{Y}$ & $B$ & $L$ \\
\hline$q_{L}=\left(V_{\mathrm{CKM}}^{\dagger} u, d\right)_{L}$ & $(3,2,1 / 6)$ & $1 / 3$ & 0 \\
$u_{R}$ & $(3,1,2 / 3)$ & $1 / 3$ & 0 \\
$d_{R}$ & $(3,1,-1 / 3)$ & $1 / 3$ & 0 \\
$\Psi_{L}$ & $(3,1,-1 / 3)$ & $-2 / 3$ & 0 \\
$\Psi_{R}$ & $(3,1,-1 / 3)$ & $-2 / 3$ & -1 \\
$\ell_{L}=(\nu, e)_{L}$ & $(1,2,-1 / 2)$ & 0 & 1 \\
$e_{R}$ & $(1,1,-1)$ & 0 & 1 \\
$N_{R}$ & $(1,1,0)$ & 0 & 1 \\
$\Phi=\left(\phi^{+}, \phi^{0}\right)$ & $(1,2,1 / 2)$ & 0 & 0 \\
$S_{1}$ & $(1,1,0)$ & 0 & -1 \\
$S_{2}$ & $(1,1,0)$ & 0 & -2 \\
$\zeta$ & $(3,1,-1 / 3)$ & $-2 / 3$ & 0 \\
$\sigma$ & $(1,1,0)$ & 1 & 0 \\
\hline \hline
\end{tabular}

very heavy right-handed singlet neutrinos, as shown in Table 1 . Whereas $N_{R}$ has $L=1$ as usual, $\Psi$ is assumed to have $B=-2 / 3$ with $\Psi_{L, R}$ having $L=0,-1$. Now the scalar singlets $S_{1}, S_{2}$ have $L=-1,-2$. Hence the terms

$-\mathcal{L}_{S}=+y_{\Psi} S_{1}^{\star} \overline{\Psi_{L}} \Psi_{R}+\frac{1}{2} y_{N}^{i} S_{2} \overline{N_{i R}^{c}} N_{i R}+\kappa S_{2}^{\star}\left(S_{1}\right)^{2}+$ H.c.

are allowed. The $y_{\Psi}$ term means that $U(1)_{L}=U(1)_{P Q}$ and its spontaneous breaking through the vacuum expectation value $\left\langle S_{1}\right\rangle \neq 0$ generates the mass of the heavy quark, $M_{\Psi}$, as well as the QCD axion because $\Psi$ is a colored fermion. The resulting axion particle is thus of the KSVZ type $[16,17]$, and the domain wall number is 1 , so it is cosmologically safe [18]. The $y_{N}$ term means that $\left\langle S_{2}\right\rangle$ generates the mass of right-handed neutirnos, $M_{N}$, but the would-be singlet majoron $[19,20]$ is now related to the axion [3] through the $\kappa$ term. This simple idea says that both the neutrino seesaw anchor scale and the axion decay constant have a common origin, so the existence of one is tied to that of the other.

The key of the present new model is the addition of $\zeta$ and $\sigma$. With only $\zeta$, the allowed terms are

$-\mathcal{L}_{\zeta}=+y_{L}^{i j} \zeta^{\star} \overline{q_{i L}} i \tau^{2} q_{j L}^{c}+y_{R}^{i j} \zeta \overline{d_{i R}^{c}} u_{j R}+y_{\zeta}^{i} \zeta^{\star} \overline{N_{i R}^{c}} \Psi_{R}+$ H.c.

These terms justify the assignment that $\Psi$ has $B=-2 / 3$, and the model at this point conserves $B$. In previous work $[21,22]$, it was shown how $B$ may be broken to $(-1)^{3 B}$. Here with the addition of $\sigma$, so that the term

$$
-\mathcal{L}_{\sigma}=+y_{\sigma}^{i} \sigma^{\star} \overline{\Psi_{L}} d_{i R}+\text { H.c. }
$$

is allowed, $B$ is broken by $\langle\sigma\rangle=v_{\sigma} \neq 0{ }^{1}$

With the spontaneous breaking of $B$ by $\langle\sigma\rangle$, a massless Nambu-Goldstone (NG) boson will appear. It may be called the 'sakharon' [1] after Andrei Sakharov [24]. Such a massless particle coupled to baryon number would be highly constrained experimentally. In this proposal, the soft term

$$
V_{\text {soft }}=-\frac{\mu^{2}}{2} \sigma^{2}+\text { H.c. }
$$

is added, which violates $B$ by two units, resulting in a massive pseudosakharon instead.

For definiteness of the model parameters, we introduce the neutrino Yukawa couplings, which realize the conventional seesaw mechanism, i.e.,

$$
\mathcal{L}_{D}=-y_{D}^{\ell j} \bar{L}_{\ell} \tilde{\Phi} N_{j R}+\text { H.c. }
$$

For later convenience, we also define the mixing between the down-type quarks and the heavy quark $\Psi$ through the mass matrix linking them, i.e.,

$$
\begin{aligned}
& \left(\begin{array}{ll}
\overline{d_{i L}} & \overline{\Psi_{L}}
\end{array}\right)\left(\begin{array}{cc}
m_{d}^{i} & 0 \\
y_{\sigma}^{i} v_{\sigma} & M_{\Psi}
\end{array}\right)\left(\begin{array}{l}
d_{i R} \\
\Psi_{R}
\end{array}\right) \\
& \quad \rightarrow\left(\begin{array}{ll}
\overline{d_{i L}} & \overline{\Psi_{L}}
\end{array}\right) U_{L}^{\dagger}\left(\begin{array}{cc}
\widehat{m}_{d}^{i} & 0 \\
0 & \widehat{M_{\Psi}}
\end{array}\right) U_{R}\left(\begin{array}{l}
d_{i R} \\
\Psi_{R}
\end{array}\right),
\end{aligned}
$$

where

$$
U_{X}(X=L, R)=\left(\begin{array}{cc}
\cos \theta_{X} & \sin \theta_{X} \\
-\sin \theta_{X} & \cos \theta_{X}
\end{array}\right)
$$

We then rename the $d$ and $\Psi$ fields as the ones in the basis of their mass eigenstates. The resulting right-handed mixing is approximately given by $\theta_{R} \approx y_{\sigma} v_{\sigma} / M_{\Psi}$, while the left-handed mixing is further suppressed, i.e., $\theta_{L} \approx m_{d} y_{\sigma} v_{\sigma} / M_{\Psi}^{2}$.

The scalar potential consisting of $\Phi, S_{1}, S_{2}$, and $\sigma$ is simply given by

\footnotetext{
${ }^{1}$ The idea that a scalar singlet carrying baryon number may have a vacuum expectation value was first proposed [23] many years ago in the context of superstring-inspired $E_{6}$ models.
} 


$$
\begin{aligned}
V= & -\mu_{\Phi}^{2} \Phi^{\dagger} \Phi-\mu_{1}^{2}\left|S_{1}\right|^{2}-\mu_{2}^{2}\left|S_{2}\right|^{2}-\mu_{\sigma}^{2}|\sigma|^{2}-\frac{\mu^{2}}{2}\left(\sigma^{2}+\text { H.c. }\right) \\
& -\kappa\left(S_{2}^{\star}\left(S_{1}\right)^{2}+\text { H.c. }\right)+\frac{\lambda_{\Phi}}{2}\left(\Phi^{\dagger} \Phi\right)^{2}+\frac{\lambda_{1}}{2}\left|S_{1}\right|^{4}+\frac{\lambda_{2}}{2}\left|S_{2}\right|^{4} \\
& +\frac{\lambda_{\sigma}}{2}|\sigma|^{4}+\lambda_{12}\left|S_{1}\right|^{2}\left|S_{2}\right|^{2}+\left(\lambda_{1 \Phi}\left|S_{1}\right|^{2}+\lambda_{2 \Phi}\left|S_{2}\right|^{2}\right. \\
& \left.+\lambda_{\Phi \sigma}|\sigma|^{2}\right)\left(\Phi^{\dagger} \Phi\right)+\left(\lambda_{1 \sigma}\left|S_{1}\right|^{2}+\lambda_{2 \sigma}\left|S_{2}\right|^{2}\right)|\sigma|^{2} .
\end{aligned}
$$

In addition to being invariant under the SM gauge symmetry, it is also invariant under $U(1)_{L}$ and $U(1)_{B}$ except for the soft $\mu^{2}$ term which breaks $U(1)_{B}$ to the baryon triality, $(-1)^{3 B}$. No other soft breaking term such as $\sigma, \sigma^{3},\left(\Phi^{\dagger} \Phi\right) \sigma$, etc., is introduced since these terms disturb the observed baryon triality relation. Let $\left\langle\phi^{0}\right\rangle=v=174 \mathrm{GeV}$ which breaks $S U(2)_{L} \times U(1)_{Y}$ to $U(1)_{Q},\left\langle S_{1}\right\rangle=f_{1}$ and $\left\langle S_{2}\right\rangle=$ $f_{2}$ which break $U(1)_{L}$, and $\langle\sigma\rangle=v_{\sigma}$ which breaks $U(1)_{B}$, then the minimum of $V$ is determined by

$$
\begin{array}{r}
-\mu_{\Phi}^{2}+\lambda_{\Phi} v^{2}+\lambda_{1 \Phi} f_{1}^{2}+\lambda_{2 \Phi} f_{2}^{2}+\lambda_{\Phi \sigma} v_{\sigma}^{2}=0 \\
-\mu_{1}^{2}-2 \kappa f_{2}+\lambda_{1} f_{1}^{2}+\lambda_{12} f_{2}^{2}+\lambda_{1 \Phi} v^{2}+\lambda_{1 \sigma} v_{\sigma}^{2}=0 \\
-\mu_{2}^{2}-\kappa\left(f_{1}^{2} / f_{2}\right)+\lambda_{2} f_{2}^{2}+\lambda_{12} f_{1}^{2}+\lambda_{2 \Phi} v^{2}+\lambda_{2 \sigma} v_{\sigma}^{2}=0 \\
-\mu_{\sigma}^{2}-\mu^{2}+\lambda_{\sigma} v_{\sigma}^{2}+\lambda_{\Phi \sigma} v^{2}+\lambda_{1 \sigma} f_{1}^{2}+\lambda_{2 \sigma} f_{2}^{2}=0
\end{array}
$$

Let

$$
\begin{array}{cc}
\Phi=\left(\begin{array}{c}
i \eta^{+} \\
v+\frac{\rho+i \eta}{\sqrt{2}}
\end{array}\right), & S_{1}=f_{1}+\frac{\rho_{1}+i \eta_{1}}{\sqrt{2}}, \\
S_{2}=f_{2}+\frac{\rho_{2}+i \eta_{2}}{\sqrt{2}}, & \sigma=v_{\sigma}+\frac{\rho_{\sigma}+i \eta_{\sigma}}{\sqrt{2}} .
\end{array}
$$

then the $2 \times 2$ mass-squared matrix spanning $\left(\eta_{1}, \eta_{2}\right)$ is given by

$$
\mathcal{M}_{\eta}^{2}=\kappa\left(\begin{array}{cc}
4 f_{2} & -2 f_{1} \\
-2 f_{1} & f_{1}^{2} / f_{2}
\end{array}\right)
$$

The massless NG boson mode corresponds to the spontaneous breaking of $U(1)_{L}$, i.e., the majoron $J$. We assume $f_{1,2} \gg v, v_{\sigma}$, hence $f=\sqrt{f_{1}^{2}+4 f_{2}^{2}}$ is the axion decay constant $F_{A}$ and must be large [25]: $F_{A}>4 \times 10^{8} \mathrm{GeV}$, and $J$ is also the QCD axion. Also from Eq. (1), $f_{2}$ determines the neutrino seesaw anchor scale. The decays of the lightest $N$ generate a lepton asymmetry which gets converted by sphalerons to the present baryon asymmetry of the Universe. The state $K$ orthogonal to $J$ is assumed to be superheavy with $M^{2}=\kappa\left(f_{1}^{2}+4 f_{2}^{2}\right) / f_{2}$ :

$$
\left(\begin{array}{l}
J \\
K
\end{array}\right)=\left(\begin{array}{cc}
f_{1} / f & 2 f_{2} / f \\
-2 f_{2} / f & f_{1} / f
\end{array}\right)\left(\begin{array}{l}
\eta_{1} \\
\eta_{2}
\end{array}\right) .
$$

A pseudo-NG boson associated with the baryon number, the pseudosakharon $S \equiv \eta_{\sigma}$, is also generated with mass given by

$$
m_{S}^{2}=2 \mu^{2} .
$$

Note that $\mu^{2}$ is the soft breaking term of the baryon number conservation. The pseudosakharon $S$ is the (long-lived) DM candidate in this model. The mass matrices for real components of the fields spanning $\left(\rho_{1}, \rho_{2}\right)$ and $\left(\rho, \rho_{\sigma}\right)$ are

$$
\begin{aligned}
\mathcal{M}_{\rho}^{2} & =\left(\begin{array}{cc}
2 \lambda_{1} f_{1}^{2} & -2 \kappa f_{1} \\
-2 \kappa f_{1} & 2 \lambda_{2} f_{2}^{2}+\kappa\left(f_{1}^{2} / f_{2}\right)
\end{array}\right), \\
\mathcal{M}^{2} & =\left(\begin{array}{cc}
2 \lambda_{\Phi} v^{2} & 2 \lambda_{\Phi \sigma} v v_{\sigma} \\
2 \lambda_{\Phi} v v_{\sigma} & 2 \lambda_{\sigma} v_{\sigma}^{2}
\end{array}\right) .
\end{aligned}
$$

The mass eigenstates are defined as

$$
\left(\begin{array}{c}
h_{125} \\
h_{\sigma}
\end{array}\right)=\left(\begin{array}{cc}
\cos \theta_{h} & \sin \theta_{h} \\
-\sin \theta_{h} & \cos \theta_{h}
\end{array}\right)\left(\begin{array}{c}
\rho \\
\rho_{\sigma}
\end{array}\right) .
$$

where $\tan 2 \theta_{h}=2 \lambda_{\Phi \sigma} v v_{\sigma} /\left(\lambda_{\Phi} v^{2}-\lambda_{\sigma} v_{\sigma}^{2}\right)$. Hereafter, we assume $\lambda_{\Phi \sigma} \ll 1$ in order to avoid the stringent constraints from the Higgs invisible decay;

$$
\Gamma\left(h_{125} \rightarrow S S\right) \approx \frac{1}{2 !} \frac{\left(\sqrt{2} \lambda_{\Phi \sigma} v\right)^{2}}{16 \pi m_{h}} \sqrt{1-\frac{4 m_{S}^{2}}{m_{h}^{2}}},
$$

and the DM direct detection search;

$$
\sigma_{D D}=\lambda_{\Phi \sigma}^{2} \frac{f_{N}^{2}}{16 \pi} \frac{\bar{m}^{2} m_{N}^{2}}{m_{S}^{2} m_{h}^{4}}
$$

where $\bar{m}=m_{S} m_{N} /\left(m_{S}+m_{N}\right)$ and $f_{N}=0.308 \pm 0.018$. Thus, $\theta_{h} \approx 2 \lambda_{\Phi \sigma} v v_{\sigma} /\left(m_{h}^{2}-m_{\sigma}^{2}\right)$, where the masses of these scalar bosons are approximately given by $m_{h_{125}}^{2} \equiv m_{h}^{2} \approx$ $2 \lambda_{\Phi} v^{2}$ and $m_{h_{\sigma}}^{2} \equiv m_{\sigma}^{2} \approx 2 \lambda_{\sigma} v_{\sigma}^{2}$.

\section{LONG-LIVED DARK MATTER AND PROTON DECAY}

The pseudosakharon $S$ can decay to $d \bar{d}$ only through the SM-heavy quark mixing $\theta_{L} \approx m_{d}^{i} y_{\sigma}^{i} v_{\sigma} / M_{\Psi}^{2}$ with rate given by

$$
\Gamma(S \rightarrow d \bar{d}) \approx \frac{m_{S}}{16 \pi}\left(y_{\sigma}^{d}\right)^{4}\left(\frac{m_{d} v_{\sigma}}{M_{\Psi}^{2}}\right)^{2} \sqrt{1-\frac{4 m_{d}^{2}}{m_{S}^{2}}} .
$$

Thus, the longevity of the DM can always be maintained by choosing small $y_{\sigma}$ as in 


$$
\begin{aligned}
\tau_{S} \simeq & 10^{27} \mathrm{sec} \times\left(\frac{M_{\Psi}}{10^{10} \mathrm{GeV}} \frac{6.5 \times 10^{-3}}{y_{\sigma}^{d}}\right)^{4} \\
& \times\left(\frac{4.7 \mathrm{MeV}}{m_{d}} \frac{20 \mathrm{GeV}}{v_{\sigma}}\right)^{2}\left(\frac{20 \mathrm{GeV}}{m_{S}}\right) .
\end{aligned}
$$

This is easily set to be greater than $10^{27}$ seconds to avoid all possible cosmological constraints on $S$ as a DM candidate. Similarly, $y_{\sigma}^{s}$ and $y_{\sigma}^{b}$ must be suppressed by the additional factor of $\sqrt{m_{d} / m_{s}}$ and $\sqrt{m_{d} / m_{b}}$ respectively.

For $S$ to be DM, the coupling $\lambda_{\Phi \sigma}$ must be small to satisfy the Higgs invisible decay [26,27] and the direct-search constraints [28]. This means that the annihilation cross section of $S$ through the SM Higgs boson to SM particles is much smaller than the canonical value of $\sigma v_{\text {rel }} \approx 1 \mathrm{pb}$ to have the correct relic abundance. However, if $m_{S}>m_{\sigma}$, the relic abundance is fixed by the $S S \rightarrow h_{\sigma} h_{\sigma}$ process instead, at temperatures below $2 m_{S}$ but above $2 m_{\sigma}$. The annihilation cross section of $S S \rightarrow h_{\sigma} h_{\sigma}$ times its relative velocity is given by

$$
\sigma v_{\mathrm{rel}} \approx \frac{1}{2 !} \frac{\lambda_{\sigma}^{2}}{32 \pi m_{S}^{2}}\left(1-\frac{3 \xi}{4-\xi}+\frac{\xi}{2-\xi}\right)^{2} \sqrt{1-\xi}
$$

where $\xi=m_{\sigma}^{2} / m_{S}^{2}$. Note that the cross section is maximized for $\xi=0$, and vanishes for $\xi=1$. The pseudosakharon remains in thermal equilibrium with the SM particles through its scattering with $h_{\sigma}$ which in turn interacts and mixes with the SM Higgs and has effective Yukawa couplings to the SM fermions. As the temperature of the Universe drops below $2 m_{S}, S$ freezes out because it is effectively stable due to its very long lifetime. As for $h_{\sigma}$, it decays quickly away so that only SM particles remain in thermal equilibrium, and the usual big bang nucleosynthesis is not disturbed. Note the crucial built-in condition that $S$ does not mix with the SM Higgs because of automatic $C P$ invariance in the scalar sector. ${ }^{2}$

In Fig. 1, the constraint from obtaining $100 \%$ of the DM relic abundance is given in the $\left(m_{S}, \lambda_{\sigma}\right)$ plane for different values of $m_{\sigma} / m_{S}$. Within the parameter space of $m_{S}, \lambda_{\sigma}$ and $m_{\sigma}$ under the condition $m_{\sigma}^{2} \approx 2 \lambda_{\sigma} v_{\sigma}^{2}$, there are clearly other allowed values.

At energy scales below the new heavy particle masses $\left(M_{\zeta}, M_{\Psi}, M_{N}\right)$, the following higher dimensional operators are generated;

$$
\mathcal{L}_{\mathrm{dim} 6}^{\mathrm{eff}}=\frac{2}{M_{\zeta}^{2}}\left|+y_{L}^{i j} \overline{q_{i L}} i \tau^{2} q_{L j}^{c}+y_{R}^{i j \star} \overline{u_{j R}} d_{i R}^{c}\right|^{2}
$$

\footnotetext{
${ }^{2}$ In our minimal framework, the scalar sector is $C P$ invariant. On the other hand, in general scalar sector, the $C P$ symmetry may be violated by introducing additional degrees of freedom, e.g., a second Higgs doublet.
}

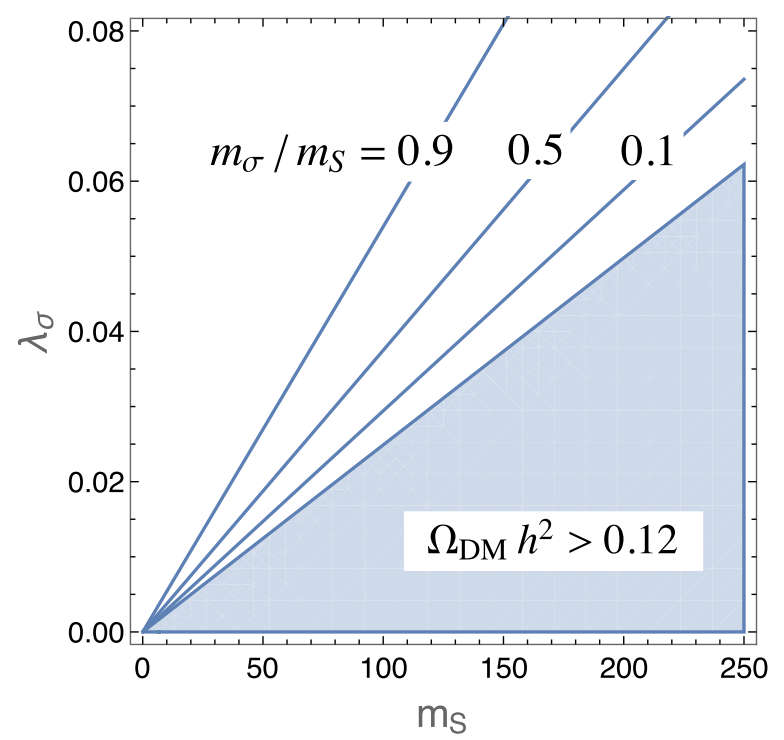

FIG. 1. Relic abundance constraints on $m_{S}$ and $\lambda_{\sigma}$ for different $m_{\sigma} / m_{S}$ values.

$$
\begin{aligned}
\mathcal{L}_{\operatorname{dim} 7}^{\text {eff }}= & \frac{2}{M_{\zeta}^{2}} \frac{y_{\zeta}^{i}\left(y_{\sigma}^{i} v_{\sigma}\right) y_{D}^{\ell i}}{M_{N}^{i} M_{\Psi}} \overline{\ell_{L}} \tilde{\Phi} d_{i R}\left(+y_{L}^{i j \star} \overline{q_{i L}^{c}} i \tau^{2} q_{L j}\right. \\
& \left.+y_{R}^{i j} \overline{u_{j R}^{c}} d_{i R}\right)+ \text { H.c. } \\
& \mathcal{L}_{\operatorname{dim} 8}^{\text {eff }}=\frac{2}{M_{\zeta}^{2}}\left|\frac{y_{\zeta}^{i}\left(y_{\sigma}^{i} v_{\sigma}\right) y_{D}^{\ell i}}{M_{N}^{i} M_{\Psi}} \overline{\ell_{L}} \tilde{\Phi} d_{i R}\right|^{2} .
\end{aligned}
$$

The dimension-six four quark operators are constrained by the LHC data. Parametrizing the coefficient of the dimension-six operators by $(2 \pi) / \Lambda^{2}$, the lower bound on this contact interactions is about 12.8 (17.5) $\mathrm{TeV}$ depending on the sign of the operators [29]. This bound is easily evaded by choosing heavier diquark with smaller Yukawa couplings. Although the dimension-six operators are the new source of the quark contact interactions at the tree level, there are no treelevel contributions to meson mixing.

Although both baryon number and lepton numbers are broken, the dimension-seven operators induce the $B+L$ conserving proton decay in Fig. 2 as in some previous proposals [30-32]. The dominant decay is $p \rightarrow \pi^{+} \nu$ and not the usual $p \rightarrow \pi^{0} e^{+}$, where the latter conserves $B-L$. Since the Higgs field is replaced by its VEV at low energy, the effective operators relevant for the proton decay are given by

$$
\mathcal{L}_{p \rightarrow \pi^{+} \nu}=C_{L} \mathcal{O}_{L}+C_{R} \mathcal{O}_{R}+\text { H.c. }
$$

where

$\mathcal{O}_{L}=\left(\overline{q_{i L}^{c}} i \tau^{2} q_{L j}\right)\left(\overline{\nu_{L}} d_{R}\right), \quad \mathcal{O}_{R}=\left(\overline{u_{j R}^{c}} d_{i R}\right)\left(\overline{\nu_{L}} d_{R}\right)$, 


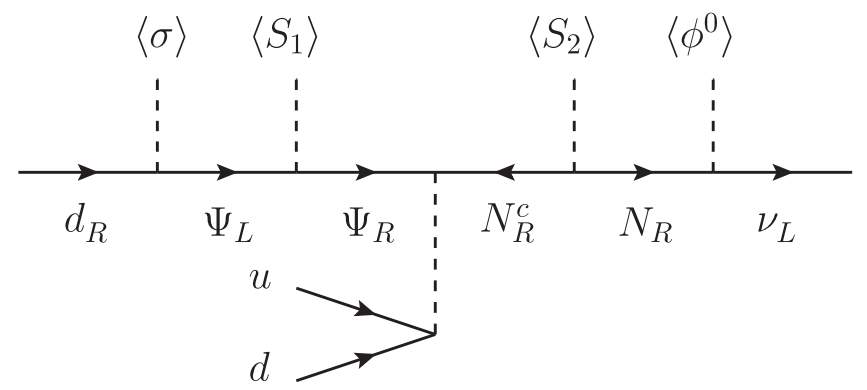

FIG. 2. Proton decay induced by $\Psi, N_{R}$, and $\zeta$.

$$
\begin{aligned}
C_{L} & =-\frac{2}{M_{\zeta}^{2}} \frac{y_{\zeta}^{i}\left(y_{\sigma}^{i} v_{\sigma}\right)\left(y_{D}^{\ell i} v\right)}{M_{N}^{i} M_{\Psi}} y_{L}^{i j \star}, \\
C_{R} & =-\frac{2}{M_{\zeta}^{2}} \frac{y_{\zeta}^{i}\left(y_{\sigma}^{i} v_{\sigma}\right)\left(y_{D}^{\ell i} v\right)}{M_{N}^{i} M_{\Psi}} y_{R}^{i j} .
\end{aligned}
$$

The proton decay rate is calculated from this effective Lagrangian as

$$
\begin{aligned}
\Gamma\left(p \rightarrow \pi^{+} \nu\right)= & \frac{m_{p}}{32 \pi}\left(1-\frac{m_{\pi}^{2}}{m_{p}^{2}}\right)^{2} \mid C_{L}\left\langle\pi^{+}\left|(u d)_{L} d_{R}\right| p\right\rangle \\
& +\left.2 C_{R}\left\langle\pi^{+}\left|(u d)_{R} d_{R}\right| p\right\rangle\right|^{2} .
\end{aligned}
$$

Thanks to the parity symmetry in $\mathrm{QCD},\left\langle\pi^{+}\left|(u d)_{\Gamma} d_{R}\right| p\right\rangle=$ $\left\langle\pi^{+}\left|(u d)_{\Gamma} d_{L}\right| p\right\rangle(\simeq 0.18)$, which are given in Ref. [33]. Then the proton lifetime is evaluated as

$$
\begin{aligned}
\tau_{p \rightarrow \pi^{+} \nu} \simeq & 8 \times 10^{32} \mathrm{yr} \times\left(\frac{M_{\zeta}}{3 \mathrm{TeV}}\right)^{4}\left(\frac{M_{\Psi}}{10^{10} \mathrm{GeV}} \frac{20 \mathrm{GeV}}{v_{\sigma}}\right)^{2} \\
& \times\left(\frac{M_{N}}{10^{10} \mathrm{GeV}} \frac{10^{-10} \mathrm{GeV}}{m_{\nu}}\right) \\
& \times\left(\frac{0.1}{y_{\zeta}} \frac{3.3 \times 10^{-3}}{y_{\sigma}}\right)^{2}\left(\frac{y_{L}}{10^{-2}} \frac{\left\langle\pi^{+}\left|(u d)_{L} d_{R}\right| p\right\rangle}{0.18}\right. \\
& \left.+2 \frac{y_{R}}{10^{-2}} \frac{\left\langle\pi^{+}\left|(u d)_{R} d_{R}\right| p\right\rangle}{0.18}\right)^{-2} .
\end{aligned}
$$

The current lower limit on this mode would be the same as that of $B-L$ conserving decay mode, i.e., $\tau_{p \rightarrow \pi^{+} \bar{\nu}}>3.9 \times$ $10^{32} \mathrm{yr}$ [34]. The observable proton decay may be within reach in future experiments. Note that the longevity of the proton is now linked to the longevity of DM and also the smallness of the neutrino mass.

\section{SUMMARY}

We have constructed a model which connects the proton and DM longevity and the smallness of neutrino mass. In our new proposal, the lepton number symmetry $(L)$ is identified as the PQ symmetry in the KSVZ model together with the conventional seesaw mechanism, where the pseudo-NG boson associated with the lepton number symmetry breaking behaves as the QCD axion. We have defined the lepton number of the heavy colored fermion $\Psi$ by introducing two new scalar fields $S_{1}$ and $S_{2}$ whose lepton numbers are different. At the same time, the uniquely defined baryon number $(B)$ is assigned to $\Psi$ through the diquark $\zeta$. We then introduced a new scalar $\sigma$ charged under $B$. The spontaneous breaking of $B$ by $\langle\sigma\rangle$ as well as an explicit soft violating term result in the pseudo-NG boson, dubbed the pseudosakharon, which is identified as the new long-lived DM, with $B$ broken to $(-1)^{3 B}$. Consequently, this model predicts the dominant $B+L$ conserving proton decay, i.e., $p \rightarrow \pi^{+} \nu$ and not the usual $p \rightarrow \pi^{0} e^{+}$which conserves $B-L$. This new connection between the DM longevity and the proton longevity opens up a new understanding of possible long-lived DM.

\section{ACKNOWLEDGMENTS}

This work was supported in part by the U.S. Department of Energy Grant No. DE-SC0008541 (E. M.) and by JSPS KAKENHI Grant No. 16K17697 (K. T.). E. M. thanks Kyoto University for support during a recent visit.
[1] E. Ma, Mod. Phys. Lett. A 32, 1730007 (2017).

[2] J. E. Kim, Phys. Rev. D 24, 3007 (1981).

[3] M. Shin, Phys. Rev. Lett. 59, 2515 (1987); 60, 383(E) (1988).

[4] E. Ma, Phys. Lett. B 514, 330 (2001).

[5] E. Ma, Phys. Rev. D 88, 117702 (2013).

[6] M. Fukugita and T. Yanagida, Phys. Lett. B 174, 45 (1986).

[7] R. D. Peccei and H. R. Quinn, Phys. Rev. Lett. 38, 1440 (1977); Phys. Rev. D 16, 1791 (1977).

[8] S. Weinberg, Phys. Rev. Lett. 40, 223 (1978).
[9] F. Wilczek, Phys. Rev. Lett. 40, 279 (1978).

[10] B. Dasgupta, E. Ma, and K. Tsumura, Phys. Rev. D 89, 041702 (2014),

[11] E. Ma, D. Restrepo, and Ó. Zapata, Mod. Phys. Lett. A 33, 1850024 (2018).

[12] E. Ma, T. Ohata, and K. Tsumura, Phys. Rev. D 96, 075039 (2017).

[13] T. R. Slatyer and C. L. Wu, Phys. Rev. D 95, 023010 (2017).

[14] Y. Mambrini, S. Profumo, and F. S. Queiroz, Phys. Lett. B 760, 807 (2016). 
[15] E. Ma, N. Pollard, R. Srivastava, and M. Zakeri, Phys. Lett. B 750, 135 (2015).

[16] J. E. Kim, Phys. Rev. Lett. 43, 103 (1979).

[17] M. A. Shifman, A. I. Vainshtein, and V. I. Zakharov, Nucl. Phys. B166, 493 (1980).

[18] P. Sikivie, Phys. Rev. Lett. 48, 1156 (1982).

[19] Y. Chikashige, R. N. Mohapatra, and R. D. Peccei, Phys. Lett. 98B, 265 (1981).

[20] G. B. Gelmini and M. Roncadelli, Phys. Lett. 99B, 411 (1981).

[21] E. Ma, Phys. Rev. D 78, 047701 (2008).

[22] E. Ma, Mod. Phys. Lett. A 24, 1335 (2009).

[23] E. Ma, Phys. Rev. Lett. 60, 1363 (1988).

[24] A. D. Sakharov, Pis'ma Zh. Eksp. Teor. Fiz. 5, 32 (1967) [JETP Lett. 5, 24 (1967)]; Usp. Fiz. Nauk 161, 61 (1991) [Sov. Phys. Usp. 34, 392 (1991)].

[25] G. Raffelt and D. Seckel, Phys. Rev. Lett. 60, 1793 (1988).
[26] M. Aaboud et al. (ATLAS Collaboration), Phys. Lett. B 776, 318 (2018).

[27] CMS Collaboration, CMS-PAS-HIG-17-023, http:// inspirehep.net/record/1662542.

[28] E. Aprile et al. (XENON Collaboration), Phys. Rev. Lett. 119, 181301 (2017).

[29] A. M. Sirunyan et al. (CMS Collaboration), arXiv:1803 .08030 .

[30] F. Vissani, Phys. Rev. D 52, 4245 (1995).

[31] K. S. Babu and R. N. Mohapatra, Phys. Rev. Lett. 109, 091803 (2012).

[32] P. H. Gu, E. Ma, and U. Sarkar, Phys. Rev. D 94, 111701 (2016).

[33] Y. Aoki, T. Izubuchi, E. Shintani, and A. Soni, Phys. Rev. D 96, 014506 (2017).

[34] K. Abe et al. (Super-Kamiokande Collaboration), Phys. Rev. Lett. 113, 121802 (2014). 